\title{
Competencias Interpersonales y Bienestar del Profesor
}

\section{Interpersonal Competence and Welfare of the Teacher}

\author{
Ángelo Serio Hernández, Heriberto Jiménez Betancort y Manuel Rosales Álamo \\ Universidad de la Laguna-España
}

\begin{abstract}
Resumen. Sin duda, gran parte del éxito escolar de los alumnos depende del bienestar de los profesores en su entorno laboral. Este trabajo se enmarca en un programa denominado IBYS (Programa de Intervención en el Bienestar y Satisfacción de los Docentes). El programa IBYS consta de 4 guías de autoformación. Cada guía trabaja diversas competencias psicoprofesionales. El objetivo de esta investigación, desarrollada con una metodología correlacional, es establecer qué competencias interpersonales caracterizan a los docentes de alto bienestar psicoprofesional y también conocer cuáles son las contra-competencias de los docentes con bajo bienestar. La muestra está configurada por 486 docentes de las Islas Canarias. Los instrumentos utilizados son el 3PD (Perfil Psicoprofesional del Docente) y el IBADA (Inventario de Bienestar de la Actividad Docente Autoevaluada). Se han alcanzado resultados significativos que muestran que las competencias interpersonales son usadas por los docentes con alto bienestar.
\end{abstract}

Palabras claves: bienestar docente, síndrome del quemado, competencias docentes, estrés laboral, competencias interpersonales.

\begin{abstract}
A large part of the school success of the pupils undoubtedly depends on the wellbeing of the teachers in their work environment. This investigation was undertaken as part of a program named IBYS (Program of Intervention in the Well-being and Satisfaction of Teachers). The IBYS program consists of 4 guides to self-training. Each guide works on various psycho-professional competences. The aim of this research, developed with a correlational methodology, is to establish what competences characterize teachers with high psychoprofessional well-being and also to learn what the counter-competences of teachers with low well-being are. The sample is formed by 486 teachers from the Canary Islands. The instruments used are 3PD (Psycho-professional Profile of the Teacher) and the IBADA (Inventory of Well-being of Educational Self-Evaluated Activity). Significant results were obtained that show that interpersonal competences are used by teachers with high well-being levels.
\end{abstract}

Key words: teacher welfare, burnout, teacher competences, job stress, interpersonal competences.

En la actualidad los problemas psicosociales representan una preocupación importante en las sociedades desarrolladas, especialmente en colectivos con baja integración social (Soriano, Franco y Justo, 2009), o en aquellas actividades laborales

La correspondencia sobre este artículo debe enviarse al primer autor al E-mail: aserio@ull.es que suponen un contacto directo con las personas objeto de la asistencia, como el campo sanitario,

\footnotetext{
Agradecimientos: La obtención de los datos utilizados en este artículo, así como la elaboración de las pruebas de diagnóstico y el propio programa que aquí presentamos, han sido posibles gracias a la subvención concedida al proyecto de Investigación IBYS por parte de la Consejería de Educación del Gobierno Canario y a los docentes y responsables de los distintos colegios de las Islas Canarias que han participado, a los que desde aquí agradecemos sinceramente su buena disposición y colaboración.
} 
policial o el educativo (Ortega y López, 2004; Aranda, Pando, Salazar, Torres y Aldrete, 2009; Moriana y Herruzo, 2005; Durán, Extremera, Montalbán y Rey, 2005). Dentro del campo educativo, las investigaciones se han focalizado en el malestar profesional o síndrome del quemado. Gran parte de estos trabajos se han centrado en detectar las fuentes de estrés (Otero-López, Castro, Villardefrancos y Santiago, 2009), en conocer los porcentajes de población afectada (CC.OO., 2000), en relacionar el malestar con aspectos de la personalidad del docente (Cebriá et al., 2001) y, por supuesto, en el diseño de programas para reconocer los factores estresores y promover el afrontamiento al estrés (García de Lucio, Camaño, Mas, Marín y García López, 2000). Aunque, sin duda, el malestar o burnout y la satisfacción docente son dos caras de una misma moneda, nuestro trabajo se desvincula del enfoque centrado en el malestar docente y se perfila en una línea de investigación mucho más positivista que, últimamente, empieza a proliferar con importantes trabajos y publicaciones, bajo el enfoque de la satisfacción o bienestar del profesorado (Lortie, 1975; Zubieta y Susinos, 1992; Marchesi, 2007). Por otro lado, también nos apartamos de la línea de estudio sobre los rasgos ya que poseen una connotación determinista como patrón de actuación que perdura a lo largo del tiempo.

Para Meijer, Korthagen y Vasalos, (2009), sigue vivo hoy en día el debate sobre si se debe trabajar la formación del profesorado desde una perspectiva más humanística del crecimiento personal del docente (Allender, 2001; Loughran, 2006). Y, por otro lado, crece el interés hacia la estandarización de competencias técnicas claramente formuladas como base para la formación del profesorado Competence Based Teacher Education- (CBTE). En esta última perspectiva se centra nuestro trabajo, en el estudio de la influencia de las competencias socio-emocionales del docente en su bienestar, salud y motivación profesional (Extremera y Fernández-Berrocal, 2003). En nuestro estudio hemos apostado por trabajar con competencias y descubrir la relación que mantienen con el bienestar psicoprofesional. Nos centramos en las competencias porque el dominio de habilidades y actitudes es mucho más moldeable o modificable. Las competencias son adquiridas por el profesorado de una forma personal, autodidacta y experiencial fundamentalmente. Sin embargo, y a pesar de ello, este tipo de habilidades pueden ser formadas o entrenadas (Vaello, 2009) y dicho cambio se puede mantener en el tiempo (Wheeler, 1999; Ciarrochi y Mayer, 2007).

Otra de las razones que nos mueve a trabajar con competencias y más concretamente de contenido socio-emocional, es la importancia que tienen en la consecución de los objetivos importantes en la vida. Cuando se pregunta a los profesores a qué habilidades propias atribuyen las metas personales conseguidas, las competencias sociales y emocionales acaparan casi todas las respuestas por encima de las competencias cognitivas. Por tanto, competencias como la fuerza de voluntad, la perseverancia, el autocontrol, el afán de superación, la capacidad de auto motivación, ocupan los primeros lugares, mientras que en pocas ocasiones los docentes mencionan aspectos como inteligencia, memoria, razonamiento, etc. Por tanto, es la madurez socio-emocional la que nos permite conseguir los logros más importantes a lo largo de la vida (Vaello, 2009).

La amplitud de las definiciones sobre el término competencia ha hecho que algunos autores hayan llegado a identificar hasta 225 modelos de competenciales distintos (Marchesi, 2007). Desde nuestra concepción de las competencias, las entendemos como un conjunto de habilidades y actitudes psicológicas que permiten al docente un desempeño personalizado y un mejor ajuste y adaptación psicoprofesional a los diferentes sucesos y experiencias de la docencia.

A pesar de las dificultades para converger o precisar mejor el concepto de competencia (Pérez Gómez, Martínez, Torres, Angulo y Álvarez, 2009), se ha hecho un esfuerzo por clasificar las distintas competencias docentes por autores como Scriven, 
1994; Angulo, 1999; o Perrenoud, 2004. Haciendo un esfuerzo de síntesis entre las propuestas de estos autores, Cano (2009), habla de competencias de organización y planificación, de comunicación, de trabajo en equipo, de relaciones interpersonales y resolución de conflictos, y de utilización de las nuevas tecnologías. Como señala Marchesi (2007), todas estas competencias son planteadas como objetivos que han de alcanzar los alumnos al término de las diferentes etapas educativas y es necesario distinguirlas de aquellas otras competencias profesionales que deben de tener los docentes para realizar bien su trabajo y conseguir un adecuado nivel de bienestar psicoprofesional. En esta última línea de trabajo se ha centrado nuestra investigación. En seleccionar las competencias interpersonales utilizadas en el programa IBYS (Jiménez, Rosales y Serio, 2008) y relacionarlas con el bienestar psicoprofesional de los docentes.

En esta línea de estudio de las competencias interpersonales como una herramienta de autoformación y bienestar docente, el dominio de las competencias socio-emocionales sirve sobre todo para conseguir mayor bienestar consigo mismo y adaptarnos mejor al escenario del aula donde pasamos la mayor parte de nuestra vida, pero por supuesto, el efecto de su dominio también repercute en la esfera de la vida familiar y social. Las nuevas propuestas educativas de cubrir objetivos competenciales en los alumnos serán difíciles de conseguir si los docentes no han sido entrenados para conseguir dichos objetivos con ellos mismos. Tienen que haber vivido las consecuencias satisfactorias del trabajo competencial en su propio bienestar psicoprofesional, para tomar conciencia de la necesidad de desarrollarlas en los demás.

Nuestro trabajo se enmarca en un proyecto mucho más amplio denominado ÓPTIMUS. Éste es un proyecto de formación, prevención, intervención e investigación del bienestar psicológico y profesional de los docentes de enseñanza no universitaria. Este proyecto ha consistido en la aplicación del programa IBYS que trabaja distintos tipos de competencias profesionales en profesores no universita- rios de las Islas Canarias (España). La organización competencial usada en nuestro programa está constituida por 4 guías auto formativas. Cada guía se compone de varios módulos de formación. Cada módulo desarrolla el aprendizaje y la sensibilización de un tipo de competencia. En esta investigación nos vamos a centrar en el estudio de la segunda guía relativa a las competencias interpersonales. En ella se trabajan en concreto las competencias promo-social, independencia, flexo-expectativa, sinergia social y disposición relacional. Nuestro trabajo presenta la novedad de introducir el término contra-competencia. Así llamamos a las formas inadecuadas de aplicar las distintas competencias o, dicho de otro modo, una contra-competencia será el conjunto de comportamientos, hábitos y actitudes psicoprofesionales que obstaculizan, interfieren o dañan el desempeño profesional de los docentes. En este artículo vamos a trabajar las contra-competencias de disociación, hostiligenia, dependencia, expectativa rígida, sinergia social y disposición relacional.

En nuestro trabajo nos planteamos las siguientes hipótesis:

1. Existe una relación significativa entre las competencias docentes estudiadas y el bienestar psicoprofesional.

2. Existe una relación significativa entre las contra-competencias docentes estudiadas y el malestar psicoprofesional.

\section{Método}

\section{Participantes}

La muestra está configurada por 486 docentes de todos los niveles de enseñanza no universitaria ( $75 \%$ mujeres y $25 \%$ varones). La distribución de la muestra en función del nivel educativo en el que trabajan los docentes aparece en la tabla 1. La media de edad de los profesores es de 42,5 años. El número total de profesores que aparece en la tabla 1 es de 509, dado que algunos ejercen en más de un nivel de docencia. 
Tabla 1. Distribución de la muestra del profesorado por nivel educativo

\begin{tabular}{lcc}
\hline Nivel de docencia & $N^{0}$ de profesores & Porcentaje \\
\hline Infantil & 79 & $15.5 \%$ \\
Primaria & 151 & $29.7 \%$ \\
Secundaria & 172 & $33.8 \%$ \\
Ciclos & 11 & $2.2 \%$ \\
Programa de garantía social & 3 & $0.6 \%$ \\
Bachillerato & 62 & $12.2 \%$ \\
Otros & 31 & $6.1 \%$ \\
\hline Total & 509 & $100 \%$ \\
\hline El número total de profesores es de 509, dado que algunos ejercen en más de un nivel de \\
docencia.
\end{tabular}

\section{Instrumentos}

Para el desarrollo de esta investigación todos los profesores participantes han ejecutado el programa IBYS (Programa de Intervención en el Bienestar y Satisfacción de los Docentes), durante unos cuatro meses aproximadamente. Este programa consta de cuatro guías. La segunda guía es la que trata las competencias interpersonales. Cada una de las guías consta básicamente de una exposición de casos donde los personajes implicados usan determinadas competencias y contra-competencias, un apartado donde se explica de forma comprensiva lo sucedido en los casos anteriores, el apartado específico donde se explican cada una de las competencias y contracompetencias y un apartado denominado "las rutas del pensamiento", donde se explica la toma de decisiones ante un conflicto y la ruta o rutas constructivas o negativas que se pueden adoptar. Después de cada uno de los apartados anteriores le sigue una sección denominada "reflexiones", donde se trata de vincular la información trabajada en la guía con la experiencia personal de los participantes. Los autores de esta investigación han elaborado dos pruebas "ad hoc". Una denominada IBADA (Inventario de Bienestar de la Actividad Docente Autoevaluada). Esta prueba recoge las valoraciones de los docentes en aspectos tales como la percepción de su eficacia docente, su motivación laboral, su relación con el alumnado, familias y compañeros y la satisfacción con su formación y con las condiciones económicas y laborales. La otra prueba utilizada en este trabajo es el 3PD (Perfil Psicoprofesional del Docente) que recoge las distintas competencias interpersonales que forman parte del programa IBYS.

\section{Procedimiento}

La metodología empleada en este estudio es de tipo correlacional. Para diferenciar a los docentes con alto y bajo bienestar psicoprofesional, se seleccionó del total de la muestra de profesores, al 25\% que tenían mayor bienestar y al $25 \%$ con peor bienestar psicoprofesional, según el IBADA.

Las competencias interpersonales, desarrolladas en el programa IBYS, hacen referencia a las distintas formas en que nos relacionamos con el alumnado, las familias y los compañeros. También se reflexiona sobre cómo percibimos a las personas y cómo ello influye en nuestra forma de interactuar con los demás. Se dividen en cuatro categorías. Las competencias psicosociales permiten la gestión de las actitudes y habilidades sociales. Las competencias de socioexpectativa permiten gestionar y optimizar lo que creemos y esperamos de los demás. Las competencias socio-profesionales hacen referencia a las habilidades que permiten a una persona tener impermeabilidad a las opiniones, comentarios y valoraciones negativas y pesimistas de los compañeros, evitando con ello contagiarse de los climas laborales carentes de atractivo y favoreciendo, a su vez, ambientes de trabajo de apoyo y cooperación entre los profesionales. Por último, trabajamos las competencias relacionales, que se refieren a las competencias que permiten a un docente positivar las relaciones interpersonales con las madres y padres de sus alumnos y alumnas.

De cada una de estas cuatro categorías competenciales se ha trabajado una competencia y una contracompetencia, excepto en la categoría de competencias psicosociales, que desarrolla dos competencias y tres contra-competencias.

A continuación presentamos las distintas competencias y contra-competencias desarrolladas en la guía 2 del programa IBYS y el significado de cada una de ellas (tabla 2). 
Tabla 2: Descripción de las competencias y contra-competencias desarrolladas en la guía 2 del programa IBYS (competencias interpersonales)

\begin{tabular}{|c|c|c|c|}
\hline Guía 2 & Competencias & ontra-competencias & Significado \\
\hline $\mathrm{C}$ & \multirow{5}{*}{ Competencias psicosociales } & Competencia promo-social & Falta de asertividad y empatía vs. asertividad y empatía. \\
\hline $\begin{array}{l}\mathrm{o} \\
\mathrm{m}\end{array}$ & & Contra-competencia disociación & Baja implicación en las relaciones con los compañeros, \\
\hline $\begin{array}{l}\mathrm{p} \\
\mathrm{e} \\
\mathrm{t}\end{array}$ & & Contra-competencia hostiligenia & $\begin{array}{l}\text { Predisposición a atacar a los demás y a defenderse para } \\
\text { combatirlos. }\end{array}$ \\
\hline $\mathrm{e}$ & & Contra-competencia & \\
\hline $\begin{array}{l}\mathrm{n} \\
\mathrm{c} \\
\mathrm{i}\end{array}$ & & dependencia vs independencia & $\begin{array}{l}\text { Dependencia vs. independencia de los demás en las rela- } \\
\text { ciones sociales. }\end{array}$ \\
\hline s & \multirow[t]{2}{*}{ Socio-expectativas } & & $\begin{array}{l}\text { Facilidad para ir cambiando las expectativas de los alum- } \\
\text { nos en función de los resultados o comportamientos. }\end{array}$ \\
\hline $\begin{array}{ll}1 \\
\mathrm{n} \\
\mathrm{t}\end{array}$ & & Contra-competencia expectativa rígida & $\begin{array}{l}\text { Dificultad para cambiar las expectativas hacia los alum- } \\
\text { nos. Se asumen como inalterables. }\end{array}$ \\
\hline $\begin{array}{l}\mathrm{r} \\
\mathrm{p}\end{array}$ & \multirow{3}{*}{ Socio-profesionales } & Competencia sinergia social & Tendencia a ser positivo, a buscar el lado positivo de los \\
\hline $\begin{array}{ll}\text { e } \\
\text { e } \\
\mathrm{r}\end{array}$ & & & $\begin{array}{l}\text { conflictos y a evitar contagiarse por la desgana o apatía } \\
\text { de los demás. }\end{array}$ \\
\hline $\begin{array}{l}\text { S } \\
\text { o } \\
\mathrm{n}\end{array}$ & & Contra-competencia disinergia & $\begin{array}{l}\text { Tendencia a desmotivar y contagiar negativamente a los } \\
\text { demás. }\end{array}$ \\
\hline $\begin{array}{l}1 \\
\mathrm{e} \\
\mathrm{s}\end{array}$ & Relacionales & $\begin{array}{l}\text { Competencia disposición relacional } \\
\text { Contra-competencia defensividad relacional }\end{array}$ & $\begin{array}{l}\text { Buena predisposición a colaborar con las familias. } \\
\text { Actitud defensiva hacia los padres de los alumnos. }\end{array}$ \\
\hline
\end{tabular}

\section{Resultados}

Tal como se aprecia en la tabla 3, los resultados obtenidos en esta investigación nos confirman que, todas las competencias mantienen relaciones significativas con el bienestar psicoprofesional de los docentes. Por otro lado, en relación a la segunda hipótesis de este trabajo, podemos decir que todas las contra-competencias guardan relaciones significativas con el bajo bienestar o malestar de los docentes. Así, guardan relación positiva con el bienestar la competencia promo-social ( $\mathrm{p} \leq .000)$, la independencia $(\mathrm{p} \leq .000)$, la flexo-expectativa $(\mathrm{p} \leq$ $.007)$, la sinergia ( $\mathrm{p} \leq .001)$ y la disposición relacional ( $\mathrm{p} \leq .000)$. A su vez, todas las contra-competencias guardan una relación positiva con el bajo bienestar psicoprofesional. La disociación ( $\mathrm{p} \leq .000)$, la hostiligenia $(\mathrm{p} \leq .000)$, la dependencia $(\mathrm{p} \leq .000)$, la expectativa rígida $(\mathrm{p} \leq .007)$, la disinergia $(\mathrm{p} \leq .001)$ y la defensividad relacional $(\mathrm{p} \leq .000)$.

Tabla 3: Diferencias de medias (t-Student) entre los docentes con alto y bajo bienestar psicoprofesional (IBADA) en las competencias interpersonales (3PD)

\begin{tabular}{|c|c|c|c|c|c|}
\hline Categoría & $\begin{array}{c}\text { Competencias y } \\
\text { Contra-competencias }\end{array}$ & $\begin{array}{l}\text { Docentes con } \\
\text { ALTO bienestar } \\
\text { psicoprofesional } \\
\text { (Media) }\end{array}$ & $\begin{array}{l}\text { Docentes con } \\
\text { BAJO bienestar } \\
\text { psicoprofesional } \\
\text { (Media) }\end{array}$ & $\mathrm{t}$ & Sig. \\
\hline \multirow{4}{*}{ Psicosociales } & Promo-social & 53.64 & 44.04 & -10.36 & .000 \\
\hline & Disociación & 5.22 & 7.90 & 6.10 & .000 \\
\hline & Hostiligenia & 10.59 & 14.83 & 6.71 & .000 \\
\hline & Dependencia vs. Independencia & 14.05 & 17.55 & 4.50 & .000 \\
\hline Socio-expectaitvas & $\begin{array}{l}\text { Flexo-expectativa vs. } \\
\text { Expectativa rígida }\end{array}$ & 29.63 & 27.84 & -2.70 & .007 \\
\hline Socio-profesionales & Sinergia vs. Disinergia & 16.53 & 9.77 & -3.46 & .001 \\
\hline \multirow{2}{*}{ Relacionales } & Disposición relacional & 23.83 & 22.00 & -3.79 & .000 \\
\hline & Defensividad relacional & 7.42 & 11.11 & 6.29 & .000 \\
\hline
\end{tabular}




\section{Discusión y conclusiones}

Los resultados obtenidos en este trabajo aportan apoyo empírico a las hipótesis planteadas. Todas las competencias utilizadas en esta investigación se relacionan con el bienestar psicoprofesional, con lo cual confirmamos la hipótesis primera. Por otro lado, también las contra-competencias se relacionan con la falta de bienestar o malestar docente, con lo cual confirmamos también la segunda hipótesis.

Dentro de la categoría de competencias psicosociales está la competencia promo-social y se refiere a las habilidades sociales de afabilidad, trato cordial y asertividad, pero también comprende la habilidad para entender el comportamiento de los otros. Esta competencia se relaciona de forma clara con el bienestar de los docentes. La independencia se refiere al respeto a las opiniones ajenas pero, al mismo tiempo, a no dejarse condicionar por las suposiciones de los demás. Vaello (2009) habla de las competencias interpersonales que son necesarias para mejorar la relación con los demás y dentro de ellas nombra la asertividad y la empatía como dos polos de una misma competencia necesaria para relacionarse, para saber hacer valer los propios derechos y para conectar afectivamente y ponerse en el lugar de los otros.

La forma inadecuada de afrontar las competencias psicosociales tiene tres posibilidades o contracompetencias. La primera tratada es la disociación o tendencia al aislamiento y la autosuficiencia. Cuando se usa la actitud disociativa se evitan los lazos y muestras afectivas y se tiene, por tanto, muchas dificultades para la integración en el trabajo cooperativo. La segunda contra-competencia la hemos llamado hostiligenia y consiste en dar muestras de desconfianza hacia los demás y mostrar malas intenciones hacia ellos. La tercera es la dependencia social o estar excesivamente pendiente de lo que opinan o piensan los demás, conlleva la excesiva necesidad de aceptación y aprobación de los otros. Como era de esperar todas ellas mantienen relaciones significativas con el malestar docente.

Dentro de la categoría socio-expectativas trabaja- mos la competencia de flexo- expectativa. Se refiere a la toma de conciencia sobre las expectativas que se tienen sobre los alumnos considerando que se pueden cambiar si se ejecutan acciones en ese sentido. Esas expectativas o creencias sobre los alumnos no se consideran una verdad absoluta y se flexibilizan en función de los datos o comportamientos que recibimos de ellos. Resulta curioso observar que en la bibliografía más reciente sobre las competencias del docente no se recoja ninguna que se relacione con el mantenimiento de expectativas positivas hacia los demás o la necesidad de hacer consciente las expectativas hacia los alumnos y tener la capacidad de flexibilizarlas. Dentro de las contra-competencias de socio- expectativa hemos trabajado con la expectativa rígida o expectativas que se tienen sobre los alumnos y que cuesta modificar, se asumen como inalterables, se hace uso de etiquetas y de categorías sobre las personas, estableciendo expectativas, pronósticos y creencias cerradas y negativas sobre los estudiantes. Este fenómeno denominado como efecto de la expectativa sostenida, (Andivia, 2009), impide que los profesores cambien sus expectativas sobre determinados alumnos aunque en éstos se produzca un verdadero cambio.

Dentro de las competencias socio-profesionales, la sinergia social se refiere a la competencia de rechazar o contra argumentar los mensajes negativos que recibimos habitualmente de otras personas de nuestro ámbito profesional. Esta competencia la tienen las personas que evitan contagiarse de ambientes laborales desmotivados y que dan mensajes positivos a sus compañeros. Sin duda es una competencia importante en determinados ambientes laborales descomprometidos con la labor docente. La disinergia es la contra-competencia y consiste en hacer análisis demoledoramente negativos de los demás, se acumulan informaciones y datos orientados a convencer a los demás de la inutilidad del esfuerzo y la implicación.

Dentro de las competencias relacionales, la disposición relacional se refiere a la necesaria actitud positiva que deben de tener los docentes hacia las familias de los alumnos. Tener la actitud de acerca- 
miento hacia ellas, aún en las condiciones más difíciles y considerar la relación con ellas como parte importante de nuestra labor profesional. Con esta competencia se busca un trato cordial y de colaboración y no tanto de juzgar y evaluar. En general, como se puede observar en la bibliografía sobre el tema del bienestar, la tendencia al colectivismo de las personas se relaciona con la interacción y el bienestar de las mismas (Oyserman, Coon y Kemmelmeier, 2002; Omar et al., 2009). En nuestra investigación este hecho se produce sobre todo en las relaciones positivas que se establecen entre las competencias promo-sociales y las de disposición relacional con el bienestar de los docentes.

La contra-competencia de "defensividad" relacional consiste en adoptar una actitud defensiva con respecto a las familias. Se tiende a pensar que éstas mantienen una actitud hostil hacia los docentes y la relación con ellos se percibe como un trámite enojoso. Algunas de las reacciones emocionales defensivas de las familias respecto al profesorado, como la atribución externa y la reactancia, son analizadas por Vaello (2009).

Los resultados encontrados en este trabajo validan la construcción de competencias y contra-competencias interpersonales realizadas en el programa IBYS. Por tanto, contribuye a dar validez al programa en lo que a competencias interpersonales se refiere.

\section{Referencias}

Andivia, L. (2009). Las expectativas de los profesores, padres y alumnos en E.S.O. Revista digital de innovación y experiencias educativas, 25, 1-8.

Angulo, F. (1999). Entrenamiento y coaching: los peligros de una vía revitalizada. En P.A. Barquín y J. Ángelo (Eds.). Desarrollo profesional del docente: Política investigación y práctica (pp.467-505). Madrid: Akal.

Allender, J.S. (2001). Teacher self: The practice of humanistic education. Lanham: Rowman \& Littlefield.
Aranda, C., Pando, M., Salazar, J.G., Torres, T.M. y Aldrete, G. (2009). Social support, burnout síndrome and occupational exhaustion among mexican traffic police agents. The spanish journal of psychology. 12, 585-592.

Cano, E. (2009). Cómo mejorar las competencias de los docentes: Guía para la autoevaluación y el desarrollo de las competencias del profesorado. Barcelona: Graó.

CC.OO. (2000). La salud laboral docente en la enseñanza pública. Federación de enseñanza de Comisiones Obreras.

Cebriá, J., Segura, J., Corbella, S., Sos, P., Comas, O., García, M., Rodríguez, C., Pardo, M.J. y Pérez, J. (2001). Rasgos de personalidad y burnout en médicos de familia. Atención primaria, 27, 39-51.

Ciarrochi, J. y Mayer, J.D. (2007). Applying emotional intelligence: a practitioner's guide. New York: Psychology Press.

Durán, M.A., Extremera, N., Montalbán, F.M. y Rey, L. (2005). Engagement y burnout en el ámbito docente: Análisis de sus relaciones con la satisfacción laboral y vital en una muestra de profesores. Revista de Psicología del trabajo y de las organizaciones 21, 145-158.

Extremera, N. y Fernández-Berrocal, P. (2003). La inteligencia emocional en el contexto educativo: hallazgos científicos de sus efectos en el aula. Revista de Educación, 332, 97-116.

García de Lucio, L., Camaño, M.D., Mas, B., Marín, M.T. y García López, F.J. (2000). Training programme in techniques of self-control and communication skills to improve nurses' relationships with relatives of seriously ill patients: a randomized controlled study. Journal of Advanced Nursing 32, 425-430.

Jiménez, H., Rosales, M. y Serio, A. (2008). IBYS: Programa de intervención en el bienestar y la satisfacción docente. Competencias interpersonales. Tenerife: Ed. Consejería de Educación del Gobierno de Canarias.

Lortie, D. (1975). The school teacher. A sociological study. Chicago: The University of Chicago Press. 
Loughran, J. (2006). Developing a pedagogy of teacher education: Understanding teaching and learning about teaching. London: Routledge.

Marchesi, A. (2007). Sobre el bienestar de los docentes: competencias, emociones y valores. Madrid: Alianza editorial.

Meijer, P.C., Korthagen, F.A y Vasalos, A. (2009). Supporting presence in teacher education: The connection between the personal and professional aspects of teaching. Teaching and Teacher Education. 25, 297-308.

Moriana, J.A. y Herruzo, J. (2005). El síndrome de burnout como predictor de bajas laborales de tipo psiquiátrico. Clínica y Salud, 16, 161-172.

Omar, A., Paris, L., Aquiar, M., Almeida, S.H. y del Pino R. (2009). Validación del inventario de bienestar subjetivo con muestras de jóvenes y adolescentes argentinos, brasileños y mexicanos. Suma Psicológica 16(2), 69-84.

Ortega, C. y López, F. (2004). El burnout o síndrome de estar quemado en los profesionales sanitarios: revisión y perspectivas. International Journal of Clinical and Health Psychology, 4, 137-160.

Otero-López, J.M., Castro, C., Villardefrancos, E. y Santiago, M.J. (2009). Job dissatisfaction and burnout in secondary schools teachers: student's disruptive behavior and conflict management examined. European Journal of Educational and
Psychology 2(2), 100-111.

Oyserman, D., Coon, H.M. y Kemmelmeier, M. (2002). Rethinking individualism and collectivism: evaluation of theoretical assumptions and meta-analyses. Psychological Bulletin, 128, 3-72.

Pérez Gómez, A.I., Martínez, J.B., Torres, J., Angulo, F. y Álvarez, J.M. (2009). Educar por competencias, ¿qué hay de nuevo? Comp. Gimeno Sacristán. Madrid: Morata.

Perrenoud, P. (2004). Diez nuevas competencias para enseñar. Barcelona: Graó.

Scriven, M. (1994). Duties of the teacher. Journal of personal evaluation in educa-tion, 8, 151-184.

Soriano, E., Franco, C. y Justo, E. (2009). Reducing psychological distress in immigrants living is Spain through the practice of flow meditation. European Journal of Educational and Psychology 2, 223-233.

Vaello, V.O. (2009). El profesor emocionalmente competente: Un puente sobre "aulas" turbulentas. Barcelona: Graó.

Wheleer, J.V. (1999). The impact of social environment on self-direct change and learning. Unpublished Doctoral Dissertation, Case Western Reserve University.

Zubieta, J.C. y Susinos, T. (1992). Las satisfacciones e insatisfacciones de los enseñantes. Madrid: CIDE.

Manuscrito recibido: $17 / 07 / 2010$

Revisión recibida: 01/09/2010

Manuscrito aceptado: 21/10/2010 